\title{
Análisis bibliométrico de los artículos científicos publicados en Medicina Veterinaria y Zootecnia en Colombia 2010-2019
}

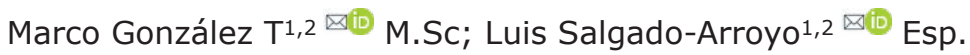

${ }^{1}$ Universidad de Córdoba. Facultad de Medicina Veterinaria y Zootecnia. Instituto de Investigaciones Biológicas del Trópico (IIBT). Montería. Colombia.

2Universidad de Antioquia. Escuela Interamericana de Bibliotecología. Medellín, Colombia.

Correspondencia: mgonzalezt@correo.unicordoba.edu.co

Recibido: Mayo 2020; Aceptado: Agosto 2020; Publicado: September 2020.

\section{RESUMEN}

Objetivo. Analizar la producción científica en Medicina Veterinaria y Zootecnia en Colombia en el período 2010-2019. Materiales y métodos. Se realizó un estudio descriptivo transversal retrospectivo, mediante el análisis bibliométrico de los artículos publicados en nueve revistas del área de la Medicina Veterinaria y Zootecnia en Colombia. La información se obtuvo mediante la búsqueda en internet de la página web de cada revista. Los artículos incluidos fueron artículos originales, revisiones de literatura, comunicaciones breves, casos clínicos y editoriales. Resultados. El total de artículos fue $3.000 ; 2.250$ (75\%) publicados en español, $673(22.4 \%)$ en inglés y $77(2.6 \%)$ en portugués. $2.282(76.1 \%)$ fueron originales, $224(7.5 \%)$ revisiones de literatura, $165(5.5 \%)$ casos clínicos, 105 (3.5\%) comunicaciones breves, 204 (6.8\%) editoriales y $21(0.6 \%)$ otros. Hubo 10.296 autores; $7.109(69 \%)$, nacionales y 3.187 (31\%) extranjeros. Del total de autores, $6.659(64.7 \%)$ fueron hombres; 3.199 (31\%) mujeres y 438 (4.3\%) sin identificación; con un promedio de 3.38 autores/artículo. Las entidades públicas aportaron el $77.9 \%$ de la producción científica y las privadas el $22.1 \%$. Las áreas con mayor número de publicaciones fueron zootecnia, salud animal, agricultura y fauna silvestre. El top 5 de las filiaciones de los autores correspondió a universidades públicas. Conclusiones. Las revistas que publican en inglés consiguen un mejor posicionamiento internacional y en ellas publican el mayor número de autores extranjeros. Los artículos más publicados fueron los originales. El top 10 de los artículos más citados arrojó un total de 810 citas que contribuyen con la internacionalización de las revistas colombianas y de Colombia.

Palabras clave: Análisis de la información; publicación científica; veterinaria; divulgación científica (Fuente: EuroVoc).

\section{ABSTRACT}

Objective. Analyze the scientific production in Veterinary Medicine and Animal Husbandry in Colombia in the period 2010-2019. Materials and methods. A retrospective cross-sectional descriptive study was carried out, through the bibliometric analysis of articles published in nine journals in the area of Veterinary Medicine and Animal Husbandry in Colombia. The information was obtained by searching the

Como citar (Vancouver).

González TM, Salgado-Arroyo LC. Análisis bibliométrico de los artículos científicos publicados en Medicina Veterinaria y Zootecnia en Colombia 2010-2019. Rev MVZ Córdoba. 2020; 25(3):e2114. https://doi.org/10.21897/rmvz.2114 
internet for each journal's website. The articles included were original articles, literature reviews, brief communications, clinical cases and editorials. Results. The total number of articles was 3.000; 2.250 (75\%) published in Spanish, 673 (22.4\%) in English and $77(2.6 \%)$ in Portuguese. $2.282(76.1 \%)$ were original, $224(7.5 \%)$ literature reviews, 165 (5.5\%) clinical cases, 105 (3.5\%) brief communications, $204(6.8 \%)$ editorials and $21(0.6 \%)$ others. There were 10,296 authors; $7.109(69 \%)$, nationals and $3.187(31 \%)$ foreigners. Of the total Authors, $6.659(64.7 \%)$ were men; $3.199(31 \%)$ women and $438(4.3 \%)$ without identification, with an average of 3.38 authors/article. Public entities contributed $77.9 \%$ of scientific production and private entities $22.1 \%$. The areas with the highest number of publications were zootechnics, animal health, agriculture and wildlife. The top 5 of affiliations of the authors corresponded to public universities. Conclusions. The journal that publish in English achieve a better international positioning and in them the largest number of foreign authors publishes. The most published articles were the original ones. The top 10 of the most cited articles yielded a total of 810 citations that contribute to the internationalization of Colombia and Colombian journals.

Keywords: Information analysis; scientific press; veterinary; popularising science (Source: EuroVoc).

\section{INTRODUCCIÓN}

Las revistas científicas se constituyen en el principal medio de difusión y divulgación de los resultados de investigación científica, y velan por la calidad de lo publicado mediante un proceso de evaluación, el cual se adapta tanto a las necesidades de cada revista, como al contexto de las comunidades científicas y, por tanto, son responsables de la circulación del conocimiento y de la contribución al desarrollo de la sociedad (1). Según López-Ornelas y Cordero-Arroyo (2), todas estas acciones se trasforman en visibilidad para los autores y para las organizaciones o instituciones a las que ellos pertenecen, por lo que las revistas se han venido utilizando como una herramienta importante para la evaluación tanto de investigadores, como de las mismas instituciones a las que pertenecen. Debido al auge que ha tenido el desarrollo tecnológico y en particular desde la aparición del internet en los 90, comenzaron a desarrollarse nuevas formas de difusión y divulgación del conocimiento. En ese contexto se pueden mencionar las revistas electrónicas, bases de datos, repositorios, bibliotecas virtuales, índices, rankings, entre otras innovaciones; lo que ha hecho que la evaluación cada día sea más compleja, debido entre otros, a nuevos factores como la usabilidad, descargas, cooperación institucional, impacto de las publicaciones, impacto del autor, índice de colaboración y altmetrics, por ejemplo (2).

En concordancia con lo descrito anteriormente, las revistas científicas se han convertido en uno de los principales objetos de estudios de la bibliometría, justamente con la intensión de que se reconozca la labor de los investigadores, a través de sus publicaciones científicas, por lo que el aspecto esencial de la bibliometría lo constituye la citación, lo que implica que no sólo es importante realizar investigaciones de buena calidad y pertinentes, sino que deben estar a disposición de la comunidad científica y por tanto, deben aportar al desarrollo de la sociedad (3). La bibliometría ha tenido un auge reciente, pero fue creada inicialmente para estudiar el comportamiento de la ciencia a través de las publicaciones, y para su desarrollo se consideró que la información bibliográfica contenía los elementos necesarios para generar un análisis con métodos matemáticos en un corto plazo (4).

En las últimas décadas, de acuerdo con Arévalo, Cordón-García y Maltrás (5), la producción científica ha aumentado vertiginosamente, así como también su indización en diferentes bases de datos, sistemas de referenciación y redes académicas, lo que ha permitido automatizar y potenciar el uso de la bibliometría. Concomitantemente se han generado indicadores para medir la evolución de la actividad científica, y que pueden ser obtenidos empleando métodos cuantitativos, basados en indicadores matemáticos para describir su progreso; así, editores e investigadores han recurrido al uso de indicadores de impacto para comprender mejor estas conductas, que por supuesto, son variables. Mediante estos indicadores se pueden evaluar de forma objetiva y seguir las tendencias de la información que generan las revistas y otras publicaciones periódicas, lo que se torna útil para generar estrategias orientadas a mejorar la gestión editorial para incrementar su impacto $(6,7)$.

Por otro lado, son frecuentes los debates sobre las limitaciones y usos apropiados para los indicadores de rendimiento de una revista, que son casi tan largos como la historia misma de las mediciones bibliométricas. Dado que la cienciometría se realiza utilizando técnicas bibliométricas, la historia de la primera está 
indisolublemente unida a la segunda. Como cualquier controversia, es difícil separar una invención de su historia, y por estas razones, se esboza una visión general de algunos eventos históricos que han sido clave para el factor de impacto. Cuando Garfield propuso el concepto hace más de 60 años (8), comenta Smith (9), que probablemente el no vislumbró que ese impacto, algún día, sería objeto de una controversia universal aún vigente.

La asociación positiva que existe entre la evaluación de los artículos por pares y el impacto, medido por el número de citas, se ha incrementado sustancialmente en los últimos años (10). Con el uso de esta información y de algunas herramientas bibliométricas, los editores tienen a su disposición elementos adicionales en que apoyarse para la toma decisiones con miras a mejorar la visibilidad e impacto de las publicaciones que editan. Estas herramientas son una propuesta de indicadores que dan cuenta de las dinámicas propias de las comunidades científicas y del conocimiento generado por estas, hoy llamadas métricas responsables o métricas alternativas (MA), y que desde su aparición han evolucionado constantemente $\mathrm{y}$, por tanto, se encuentran diferentes definiciones de acuerdo con el estudio realizado. Las Altmetrics o métricas alternativas, son una clase amplia de estadísticas que analizan el impacto de la investigación a través de medios no tradicionales o convencionales de la evaluación de la ciencia $(11,12)$. Las fuentes para obtener los datos altmétricos incluyen: servicios de microblogging o mensajes cortos (Twitter), sitios de redes sociales (Facebook), blogs (WordPress, Blogger), redes de marcadores sociales (Delicious), plataformas de marcadores académicos (CiteULike, Mendeley), servicios de revisión por pares (F1000, ahora F1000Prime), redes académicas (Academia. edu), y enciclopedias editadas en colaboración en línea (Wikipedia), (12).

De manera general y compresible para cualquier persona, se puede afirmar que son un conjunto de indicadores alternativos para medir el impacto de las publicaciones a nivel de artículo o de autor, pero a través de la Web 2.0 (Facebook, Twitter, blogs, redes académicas, etc.), (13).

Las redes sociales registran una gran cantidad de información, que una vez identificada, organizada y analizada adecuadamente, se puede utilizar para estudiar la divulgación de la comunicación científica y sus procesos en mayor profundidad. Jason Priem acuñó el término altmetrics, a través de un tuit, el 29 de septiembre de 2010, un mes más tarde se creó el Manifiesto Altmetrics para definir el estudio evaluativo de la investigación, pero analizando los productos de las herramientas académicas en línea $(14,15)$.

Los estudios bibliométricos también permiten examinar retrospectivamente un área determinada del conocimiento, evaluar el potencial de las investigaciones de las instituciones involucradas, así como también la caracterización del desarrollo de disciplinas científicas y sus líneas de investigación. Igualmente, posibilita conocer la obsolescencia y la dispersión de las publicaciones científicas; estudios que son utilizados para la formulación de políticas científicas y de gestión, en la medida que el conocimiento científico se perciba como un valor estratégico, en especial, si se considera que ellos generan unos resultados útiles $(7,10)$.

En este sentido un estudio bibliométrico realizado por Crawley (16) analizó los patrones de citas de los autores que publicaron sus artículos en la American Journal of Veterinary Research (AJVR) entre los años 2001 y 2003. Crawley (16) analizó las 25.000 referencias bibliográficas de dichos artículos y las agrupó por tipo de material, fecha de publicación y frecuencia de las revistas citadas. Los títulos de las revistas consultadas por los autores se clasificaron en orden decreciente de productividad para crear una lista central de revistas que los investigadores médicos veterinarios utilizan con mayor frecuencia. La mayoría de los artículos citados fueron de revistas $(88.8 \%)$, seguidos de los libros $(9.8 \%)$ $y$, por último, la literatura gris $(1.4 \%)$.

En la facultad de medicina veterinaria y zootecnia de la universidad peruana Cayetano Heredia (FAVEZ-UPCH), Llalla et al (17) realizaron una evaluación bibliométrica de la investigación formativa en dicha facultad en el período 20122017. Para desarrollar su investigación, ellos, diseñaron un estudio observacional, descriptivo, retrospectivo, y utilizaron herramientas bibliométricas para sistematizar la información proveniente de la base de datos del banco de tesis de la FAVEZ-UPCH. Se evaluaron 195 tesis que utilizaron 6548 referencias, predominando las consultas de los artículos científicos en idioma inglés, seguidas de español. Del total de referencias, sólo el 29\% fueron consideradas actualizadas. Los animales domésticos fueron los más estudiados (43.6\%), seguidos de los silvestres $(30.3 \%)$; los caninos $(29.2 \%)$ y 
el hombre (15.4\%) fueron las especies más estudiadas. Epidemiología y salud pública (30.7\%) y la clínica veterinaria docente Cayetano Heredia $(21.0 \%)$ desarrollaron la mayor parte de las tesis y de ellas, el $23.1 \%$ produjeron artículos científicos que se publicaron en revistas nacionales $(91.2 \%)$, (17).

El estudio realizado por Bravo-Vinaja y SanzCasado (18) caracterizó a través del uso de indicadores bibliométricos, la actividad científica de las instituciones mexicanas relacionadas con las ciencias agrícolas. Se utilizó la información de los artículos publicados durante el período 19832002 en las bases de datos Agrícola, Agris, Cab Abstracts, Science Citation Index (SCI), Social Sciences Citation Index (SSCI) y Tropag \& Rural. El criterio de búsqueda fue que en la filiación de los autores se incluyera alguna institución asentada en México. Los registros se importaron a un manejador de referencias bibliográficas ProCiteTM. El total de registros fue 15736 , de los cuales $1185(7.53 \%)$ se obtuvieron de Agrícola, 2487 (15.74\%) de Agris, 9456 de CAB Abstracts (60.09\%), $168(1.06 \%)$ de Tropag \& Rural, 2449 (15.50\%) de SCI, y $10(0.64 \%)$ de SSCI (18).

El Distrito Federal y el Estado de México publicaron más de la mitad de los artículos, lo cual muestra una alta concentración de la investigación en estos dos estados. Los sectores institucionales que mostraron mayor actividad investigadora fueron las universidades públicas y los institutos o centros de investigación. Los idiomas en que se publicaron fueron el inglés y el español. La tasa media de artículos firmados en coautoría fue de $87.62 \%$; el índice de coautoría aumentó de 2.47 autores por artículo en 1983 a 4.08 en 2002 (18).

La estructura conceptual de la investigación en Ciencia Animal (CA) fue estudiada por Rodríguez-Ledesma et al (19), a través de un análisis de mapeo longitudinal y análisis de rendimiento, que permite revelar temas ocultos y su evolución conceptual en 66 años (1945 -2011). Los autores definieron seis períodos: 1945-1969, 1970-1979, 1980-1989, 19901999, 2000-2005 y 2006-2011. Se identificó que la investigación en CA se centró en diez áreas: Alimentación de animales, pequeños rumiantes, reproducción animal, producción láctea, calidad de carne, producción porcina, cría genética y animal, aves de corral, bienestar animal y factores de crecimiento. Pero también se ha orientado al bienestar animal, a la genómica, a la gestión y a la salud humana. El análisis de mapeo proporciona una visión global de investigar la evolución a través del tiempo. Sin embargo, cuando el período es muy largo, se deben abordar períodos más cortos para una mejor comprensión de las causas de esta evolución (19).

El estudio de tópicos específicos también se puede abordar mediante lo bibliometría tal como lo reportan Ríos et al (20) quienes adelantaron un estudio sobre las publicaciones hechas en Colombia sobre enfermedades infecciosas. En el estudio, los autores relacionaron 2963 publicaciones, de las cuales 2744 (92.6\%) fueron nacionales y 219 (7.4\%) lo fueron extranjeras. Las publicaciones en general abordaron las siguientes áreas: parasitología 268 (32.8\%), bacteriología y resistencia antimicrobiana 267 (32.7\%), virología $210(25.7 \%)$, micología $46(5.6 \%)$ y otros artículos relacionados con las enfermedades infecciosas 26 (3.2\%). La distribución regional (departamentos o regiones) de las publicaciones fue: Cundinamarca 239 $(29.3 \%)$, Antioquia 211 (25.8\%) Valle del Cauca $60(7.3 \%)$, Costa Caribe $52(6.4 \%)$, Santanderes $38(4.6 \%)$, Eje cafetero $31(3.8 \%)$ y Sur del país $13(1.6 \%)$. Este artículo se constituyó en el primero que analizó las publicaciones relacionadas con las enfermedades infecciosas en Colombia y se encontró, además, que la universidad pública soportó el $90 \%$ de la investigación del área de infectología en Colombia. Estos resultados no sólo son importantes para las revistas, sino también para los organismos responsables de la salud pública nacional.

Los estudios bibliométricos también son importantes para analizar las universidades. Mattar et al (22), a partir de la producción científica, colaboración internacional, calidad científica promedio, porcentaje de las publicaciones en revistas del primer cuartil SJR y radio de excelencia de las universidades colombianas, las clasificaron de acuerdo con el escalafón Iberoamericano SCImago Institutions Ranking. De ese estudio se desprendió que sólo el $6.2 \%$ de las instituciones colombianas mantienen una producción científica competitiva frente al resto de las universidades Iberoamericanas. También encontraron que las universidades colombianas con mayor productividad fueron: Nacional de Colombia, Antioquia, Andes, Valle y Javeriana. En la región Caribe fueron: Norte, Cartagena, Córdoba, Magdalena y Sucre. Estos estudios son importantes porque permiten comparar las instituciones de educación superior colombianas con otras en el ámbito internacional. Estos 
estudios permiten una mirada contextualizada $y$, este estudio en particular recomienda que las IES debieran apostarle a la calidad y, utilizar, al menos, los cinco factores que propone SCImago. Aunque los rankings no son obligatorios, ignorarlos significaría autoaislamiento en este mundo tan globalizado.

Las revistas científicas del área de ciencias veterinarias también han sido objeto de estudios bibliométricos como lo informan Carreño et al (23), quienes analizaron los indicadores bibliométricos de actividad de la Revista MVZ Córdoba de 14 años (1994-2008) y De La Ossa et al (24), quienes realizaron un análisis bibliométrico de la producción científica publicada en la Revista Colombiana de Ciencia Animal 2009-2018.

A partir de los resultados, los autores antes citados, concluyeron que para mantener y mejorar la calidad de la revista se debía reglamentar y verificar la actualidad de las referencias, controlar la autocitación, la obsolescencia, realizar mediciones bibliométricas con mayor periodicidad y no menos importante, establecer la vigilancia permanente sobre los índices bibliométricos que se generen de las revistas para su análisis y orientaciones a las que haya lugar.

Las publicaciones relacionadas con bibliometría en el área de ciencias veterinarias en Colombia son escasas, y no se encuentran trabajos que estudien la evolución de la producción científica del área, sólo algunos artículos relacionados de la propia revista en particular. Adicionalmente, en los últimos 10 años no hay estudios sobre esta materia en específico, ni estudios conexos para establecer el estado del arte, ni siquiera parciales.

Este trabajo es una herramienta para el análisis de la comunidad científica del área de ciencias veterinarias en el país. La información aportada permitirá orientar o proponer líneas de investigación, fundamentar la toma de decisiones relacionada con las temáticas de estudio, definición de lineamientos o estrategias en la edición de las revistas científicas, repensar el idioma de la publicación, entre otros. Los objetivos específicos de la presente investigación estuvieron orientados a la determinación del volumen de la producción, establecer el grado de colaboración de autores, identificar las universidades colombianas más productivas, producciones científicas de las entidades públicas y privadas, idiomas en que se publican las revistas, género de los autores, procedencia de los autores e identificación de los artículos más citados del área.

El objetivo general del presente estudio fue analizar a través de la bibliometría la producción científica en el área de medicina veterinaria y zootecnia en Colombia en el período 2010-2019 de nueve revistas indexadas en la convocatoria IBN Publindex II - 2014 del Ministerio de Ciencia, Tecnología e Innovación y en la base de datos SciELO-Colombia.

\section{MATERIALES Y MÉTODOS}

Tipo de estudio. Se realizó un estudio descriptivo transversal retrospectivo, mediante el análisis bibliométrico de los artículos publicados en las revistas del área de Medicina Veterinaria y Zootecnia en Colombia 2010 - 2019.

Criterios de inclusión. Revistas colombianas indexadas en la convocatoria IBN Publindex II - 2014 del Ministerio de Ciencia, Tecnología e Innovación. Se consideró esta convocatoria porque fue la última que agrupó alrededor de 520 revistas antes de introducir las modificaciones al proceso de evaluación actual. De esta manera se pudo asegurar un mayor un número de revistas para el estudio. También se incluyeron las indexadas en la base de datos SciELO Colombia, ya que no todas las revistas se encuentran indexadas en otras bases de datos como Web of Science o SCOPUS.

Fuentes de información. La información general se obtuvo desde las páginas web de cada una de las revistas. La información se recopiló y filtró manualmente, visitando las páginas oficiales de cada revista, debido a que la información reportada en sus páginas principales, no se encuentra completa en las bases de datos como SciELO, SCOPUS o Web of Science. Aunque se consideró en principio incluir los análisis alternativos o altmétricos, no fue posible porque todas las revistas del estudio no utilizan DOI (Digital Object Identifier), lo que se constituye en una condición "sine qua non" para adelantarlos.

Tipos de documento considerados para el estudio. Se incluyeron las siguientes categorías: artículos originales, revisiones de literatura, comunicaciones breves, casos clínicos y editoriales que son los principales tipos de artículos que caracterizan las revistas científicas. 
Las características de los artículos seleccionados se describen a continuación:

Artículos originales. Informan sobre avances en las ciencias veterinarias con base en la investigación científica, son producto de una investigación original. Responden a una estructura general que comprende: resumen, introducción, materiales y métodos, resultados, conclusiones, discusión y referencias. Se publican en revistas que cuentan con un proceso de revisión por pares generalmente a doble ciego. Tienen una extensión de alrededor de 10 páginas.

Revisiones de literatura. Consisten en una revisión y análisis crítico de la literatura publicada en torno a un tema de la ciencia (generalmente de actualidad), de interés general y pertinencia para las ciencias veterinarias, se caracterizan por tener una sección de referencias muy extensa y tienen una extensión mayor a la de los artículos originales.

Comunicaciones breves. Tienen la misma estructura de los artículos originales, pero su extensión aproximada es de 6 páginas.

Casos clínicos. La estructura es similar al de las comunicaciones breves, pero después de la introducción se continúa con la evaluación del paciente, el enfoque del tratamiento y conclusiones.

Editoriales. Consiste en un texto expositivoargumentativo, conciso, que puede o no estar firmado, que ocupa un lugar destacado dentro de la presentación de la revista, que explica, valora y juzga un hecho noticioso, novedoso, de especial importancia para la sociedad. Recoge las contribuciones de uno o varios manuscritos publicados y generalmente finaliza con la expresión de una opinión, que puede suscitar un debate, pero también se pueden proponer nuevas estrategias de investigación, tratamientos o recomendaciones si fuere el caso.

Los artículos que no se ajustaron a las categorías descrita anteriormente, se incluyeron como otros (resúmenes de congresos, artículo de reflexión no derivado de investigación, notas cortas, obituarios, memorias, etc.).

Muestra e indicadores. El tamaño de la muestra se obtuvo a través de la suma aritmética de los artículos publicados en cada revista, teniendo en cuenta las categorías seleccionados para el estudio, a continuación, se muestran los indicadores que se tuvieron en cuenta para la recopilación de la información.
Producción científica. Fue calculada según el número de artículos indizados en cada revista

Número total de autores en el período estudiado y por revista. Correspondió a la suma aritmética del total de autores nacionales y extranjeros que publicaron en la década estudiada y desglosados a su vez por cada una de las revistas.

Origen geográfico y tipo de filiación. Se determinó la procedencia de los autores, identificando el país, la ciudad y la clasificación de la entidad de su filiación (pública, privada o mixta).

Género de los autores. El género (masculino o femenino) de los autores se identificó a través del nombre con que cada uno de ellos firmó el artículo. Se estableció una categoría como "género indeterminado" en aquellos casos en donde el nombre "per se" no permitió identificar el género (Ejemplo: M. Durán, AJ Martínez).

Índice de colaboración por revista en la década. Corresponde al cociente entre el total del número de autores y el total del número de artículos publicados por cada revista en la década estudiada.

Idiomas de publicación. Identificación de los idiomas de publicación de las revistas seleccionadas.

Área de estudio de las publicaciones. Se realizó un estudio previo (datos no mostrados) con el fin de clasificar las áreas temáticas de publicación y se identificaron las siguientes que se registran en orden alfabético: Agricultura, biotecnología, enfermedades infecciosas, ensayos veterinarios, entomología, farmacología, fauna silvestre, fitopatología, salud humana, salud animal y zootecnia. Las áreas temáticas que no se ajustaron a las descritas anteriormente, fueron ingresadas como otras.

Índice de colaboración institucional. Relación e identificación del carácter y número de instituciones filiales participantes en el artículo.

Índice de productividad. Se obtuvo mediante la aplicación del logaritmo del número de trabajos originales publicados por cada revista en la década.

Análisis de los datos. Para el almacenamiento, análisis descriptivo y análisis estadístico se utilizó el programa Excel 365 para Windows. El control de la calidad de la información se 
efectuó mediante la doble entrada de los datos, corrigiendo las inconsistencias mediante la consulta minuciosa y confrontación con las fuentes originales en tres oportunidades.

\section{RESULTADOS}

En la tabla1 se muestran las revistas seleccionadas con base en los criterios de inclusión del estudio. También se presenta la categoría en que se encontraban dichas revistas de acuerdo con los resultados de la convocatoria del IBN Publindex II-2014. Es necesario aclarar que esta es la clasificación antes que la base de datos Publindex- Minciencias (antiguo Colciencias) implementara la reforma de las políticas para la nueva medición de las revistas académicocientíficas colombianas y que involucró el ranking de SCImago (Scimago Journals Ranking). Además, se presentan los índices h5 y media h5, obtenidos directamente de la consulta en Google Scholar y Fi-SciELO; este último, capturado del sitio web de SciELO-Colombia (Scientific Electronic Library Online-Colombia).

En la tabla 2 se relaciona el total de artículos publicados por cada una de las revistas analizadas en la década 2010-2019. Se recopiló un total de 3.000 artículos para un promedio de 333.3 artículos por revista y una desviación estándar de 10.28 .

Tabla 1. Identificación y algunas características de las revistas seleccionadas.

\begin{tabular}{|c|c|c|c|c|c|}
\hline Nombre largo y abreviado de las revistas & ISSN & CIBN & H5 & Media h5 & FI SciELO \\
\hline $\begin{array}{l}\text { Revista Colombiana de Ciencias Pecuarias } \\
\text { (Rev Colomb Cienc Pecu) }\end{array}$ & $0120-0690$ & A1 & 9 & 11 & 0.1687 \\
\hline $\begin{array}{l}\text { Revista Ciencia y Tecnología Agropecuaria } \\
\text { (Cienc Tecnol Agropecuaria) }\end{array}$ & $0122-8706$ & A2 & 6 & 9 & 0.1690 \\
\hline $\begin{array}{l}\text { Revista U.D.C.A. Actualidad \& Divulgación Científica } \\
\text { (Rev UDCA Act \& Div Cient) }\end{array}$ & $0123-4226$ & $\mathrm{~A} 2$ & 11 & 13 & 0.0250 \\
\hline $\begin{array}{l}\text { Revista de Medicina Veterinaria } \\
\text { (Rev Med Vet) }\end{array}$ & 0122-9354 & $\mathrm{A} 2$ & 8 & 12 & 0.1573 \\
\hline $\begin{array}{l}\text { Revista de la Facultad de Medicina Veterinaria y de zootecnia } \\
\text { (Rev Med Vet Zoot) }\end{array}$ & 0120-2952 & $\mathrm{A} 2$ & 7 & 12 & 0.1042 \\
\hline $\begin{array}{l}\text { Revista CES Medicina Veterinaria y Zootecnia } \\
\text { (Rev CES Med Zootec) }\end{array}$ & $1900-9607$ & $\mathrm{~A} 2$ & 7 & 7 & 0.0328 \\
\hline $\begin{array}{l}\text { Revista Orinoquia } \\
\text { (Orinoquia) }\end{array}$ & 0121-3709 & A2 & 7 & 10 & 0.0938 \\
\hline $\begin{array}{l}\text { Revista Colombiana de Ciencia Animal } \\
\text { (Rev colombiana Cienc Anim - Recia) }\end{array}$ & $2027-4297$ & $\mathrm{~B}$ & 6 & 8 & 0.001 \\
\hline $\begin{array}{l}\text { Revista MVZ Córdoba } \\
\text { (Rev MVZ Córdoba) }\end{array}$ & 0122-0268 & $\mathrm{A} 1$ & 9 & 13 & 0.1963 \\
\hline
\end{tabular}

CIBN: Categoría IBN Publindex II 2014

(*) H5 y media h5 tomado de https://scholar.google.es/citations?view op=top venues\&hl=es

Tabla 2. Tipología de los artículos publicados en las revistas seleccionadas

\begin{tabular}{|c|c|c|c|c|c|c|c|}
\hline & ORI & REV & CC & CB & ED & OTR & Totales \\
\hline Rev Colomb Cienc Pecu & 269 & 36 & 19 & 16 & 39 & 2 & 381 \\
\hline Cienc Tecnol Agropecuaria & 204 & 10 & 0 & 1 & 3 & 1 & 219 \\
\hline Rev UDCA Act \& Div Cient & 454 & 24 & 14 & 2 & 21 & 0 & 515 \\
\hline Rev Med Vet & 207 & 5 & 18 & 0 & 19 & 0 & 249 \\
\hline Rev Med Vet Zoot & 132 & 8 & 22 & 0 & 28 & 0 & 190 \\
\hline Rev CES Med Zootec & 125 & 45 & 33 & 1 & 15 & 11 & 230 \\
\hline Orinoquia & 236 & 22 & 5 & 0 & 24 & 2 & 289 \\
\hline Rev Colombiana Cienc Anim - Recia & 247 & 46 & 25 & 45 & 23 & 5 & 391 \\
\hline Rev MVZ Córdoba & 408 & 28 & 28 & 40 & 32 & 0 & 536 \\
\hline Totales & $\begin{array}{c}2282 \\
(76.1 \%)\end{array}$ & $\begin{array}{c}224 \\
(7.5 \%)\end{array}$ & $\begin{array}{c}165 \\
(5.5 \%)\end{array}$ & $\begin{array}{c}105 \\
(3.5 \%)\end{array}$ & $\begin{array}{c}204 \\
(6.8 \%)\end{array}$ & $\begin{array}{c}21 \\
(0.6 \%)\end{array}$ & $\begin{array}{c}3000 \\
(100 \%)\end{array}$ \\
\hline
\end{tabular}

ORI=Originales; $\mathrm{REV}=$ Revisiones de literatura; $\mathrm{CC}=$ Casos clínicos; $\mathrm{CB}=$ Comunicaciones breves; $\mathrm{ED}=\mathrm{Editoriales;} \mathrm{OTR}=\mathrm{Otros}$. Fuente: Elaboración propia. 
De los 3000 artículos, 2.250 (75\%) se publicaron en español, $673(22.4 \%)$ en inglés y $77(2.6 \%)$ en portugués. Se encontró que la revista que más publica en español y portugués es la revista UDCA, con 469 (15.6\%) artículos publicados en español y $44(1.5 \%)$ en portugués. Por otra parte, la revista que más publica en inglés es la Revista MVZ Córdoba con 318 (10.6\%). Cabe destacar que esta revista desde el año 2014 publica en español e inglés todos los artículos y la Revista Colombiana de Ciencias Pecuarias sólo publica en idioma inglés, las demás, revistas publican en español.

Del total de artículos analizados, $2.282(76.1 \%)$ correspondieron a originales, $224(7.5 \%)$ a revisiones de literatura, 165 (5.5\%) a casos clínicos, $105(3.5 \%)$ a comunicaciones breves, $204(6.8 \%)$ a editoriales y $21(0.6 \%)$ fueron clasificados como otros, tales como obituarios, cartas al editor, resúmenes de congresos, notas cortas, etc.

En la figura 1 se observa el índice de producción y las diferencias en el número de publicaciones realizadas con relación al número de autores por artículo. Se obtuvo en promedio un 3.38 de autores por artículo. La Rev Colomb Cienc Pecu resultó con 4.09 autores por artículo y fue el mayor registro; el menor, fue para la Revista Orinoquia con 3.0.

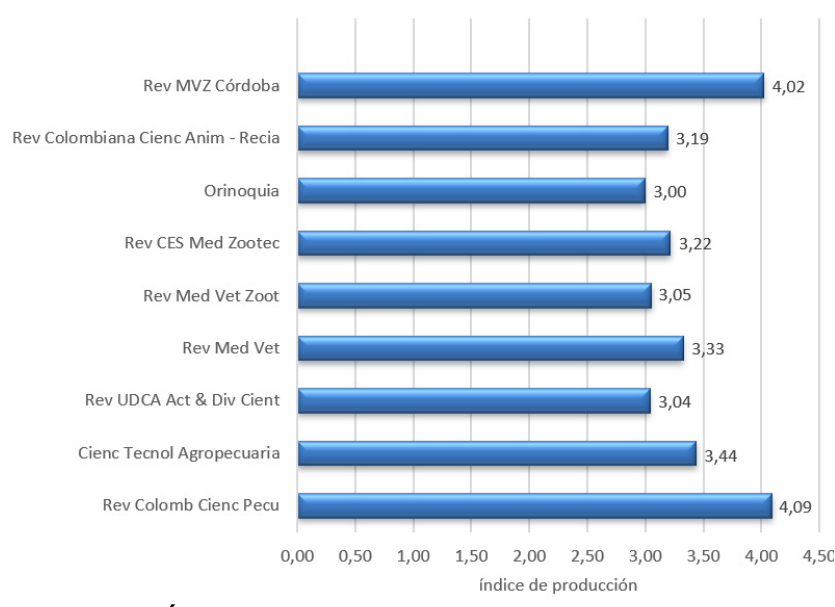

Figura 1.Índice de colaboración de la producción científica de las revistas seleccionadas.

Existe relación directa y positiva entre los autores y su productividad, con un coeficiente de Pearson de 0.950 , resultados que se pueden observar en la figura 2.

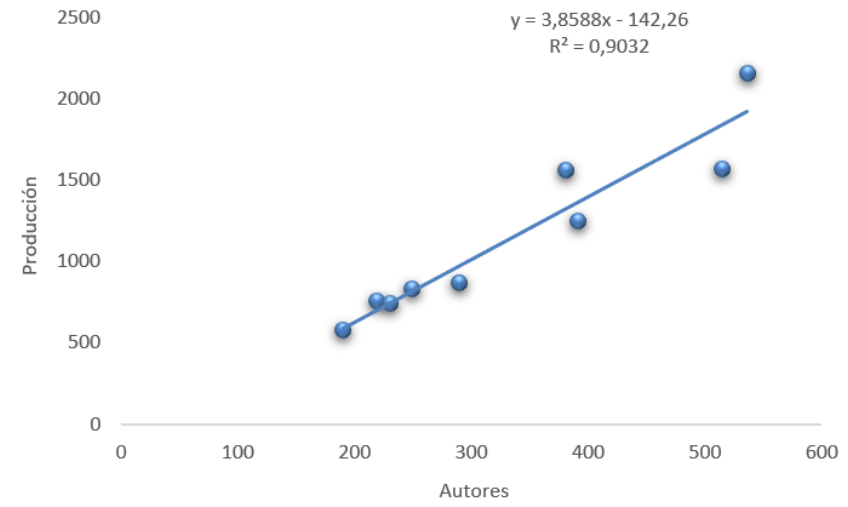

Figura 2. Coeficiente de Pearson de productividad.

Al analizar la variable autor, se encontró un total de 10.296 autores, de los cuales 7.109 (69\%), fueron nacionales y 3.187 (31\%) extranjeros (Figura 3). En general la mayoría de los autores utilizan las revistas nacionales para la publicación de sus investigaciones. Se observa que en la Revista MVZ Córdoba, así como en la Revista Colombiana de Ciencias Pecuarias, el número de autores extranjeros es mayor que los autores colombianos. Por otra parte, se identificó el género de los autores y se encontró que 6.659 $(64.7 \%)$ fueron hombres; 3.199 (31\%) mujeres y $438(4.3 \%)$ que no se pudieron identificar a través de su firma en los artículos. Por ejemplo, Martínez J; Suarez T. etc. (Figura 4).

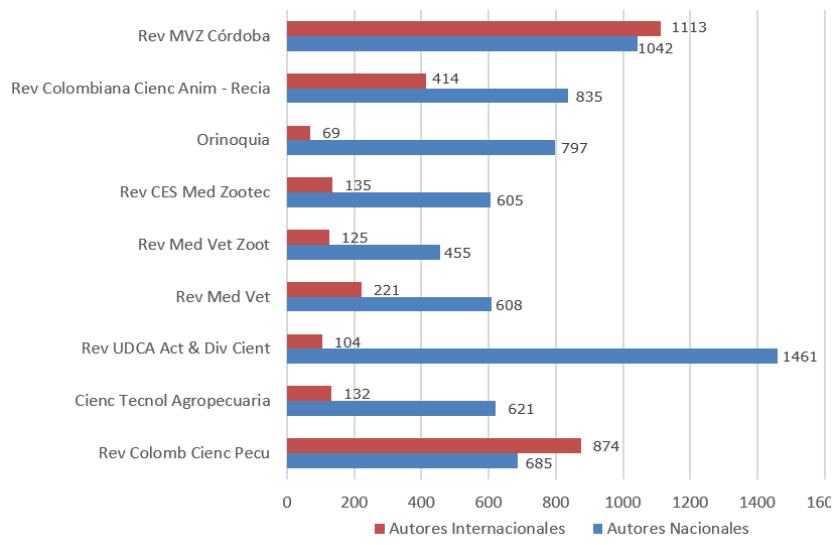

Figura 3. Procedencia de los autores en las revistas seleccionadas.

Las entidades públicas encabezaron la producción científica con $77.9 \%$ vs $22.1 \%$ de las entidades privadas. En la figura 5, se puede observar dicha relación por revista. 


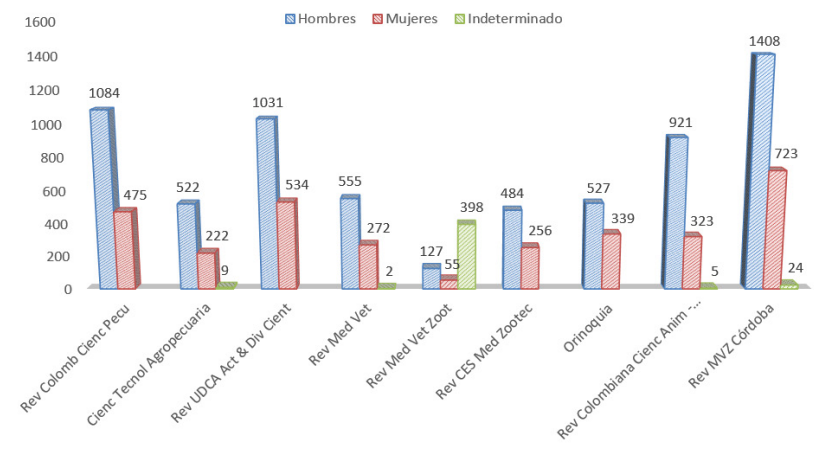

Figura 4. Género de los autores que publican en las revistas seleccionadas.

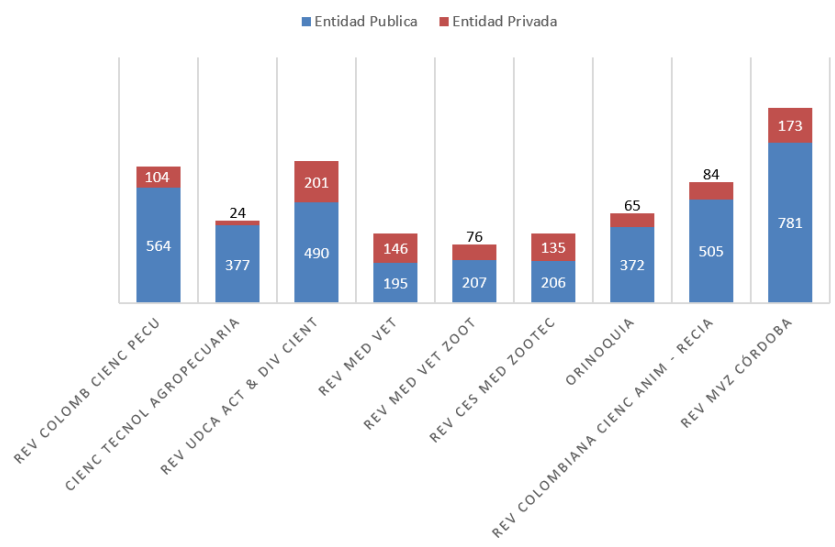

Figura 5.Participación de entidades públicas y privadas en la producción científica de las revistas seleccionadas.
En la figura 6 se puede identificar el número de autores con relación a la producción de artículos. Se destaca que tres autores es la forma más frecuente de realizar las coautorías.

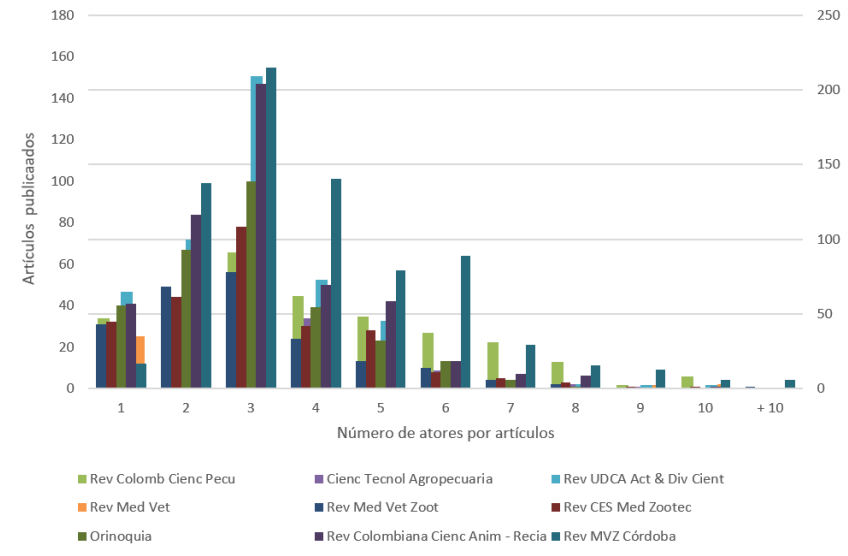

Figura 6. Número de autores que participan por artículo publicado - coautoría.

En la figura 7, se puede observar la geolocalización de los autores a nivel mundial de las revistas objeto de estudio. En esta gráfica permite inferir cierta tendencia de una creciente aceptación de las revistas colombianas por la comunidad científica mundial para publicar sus investigaciones.

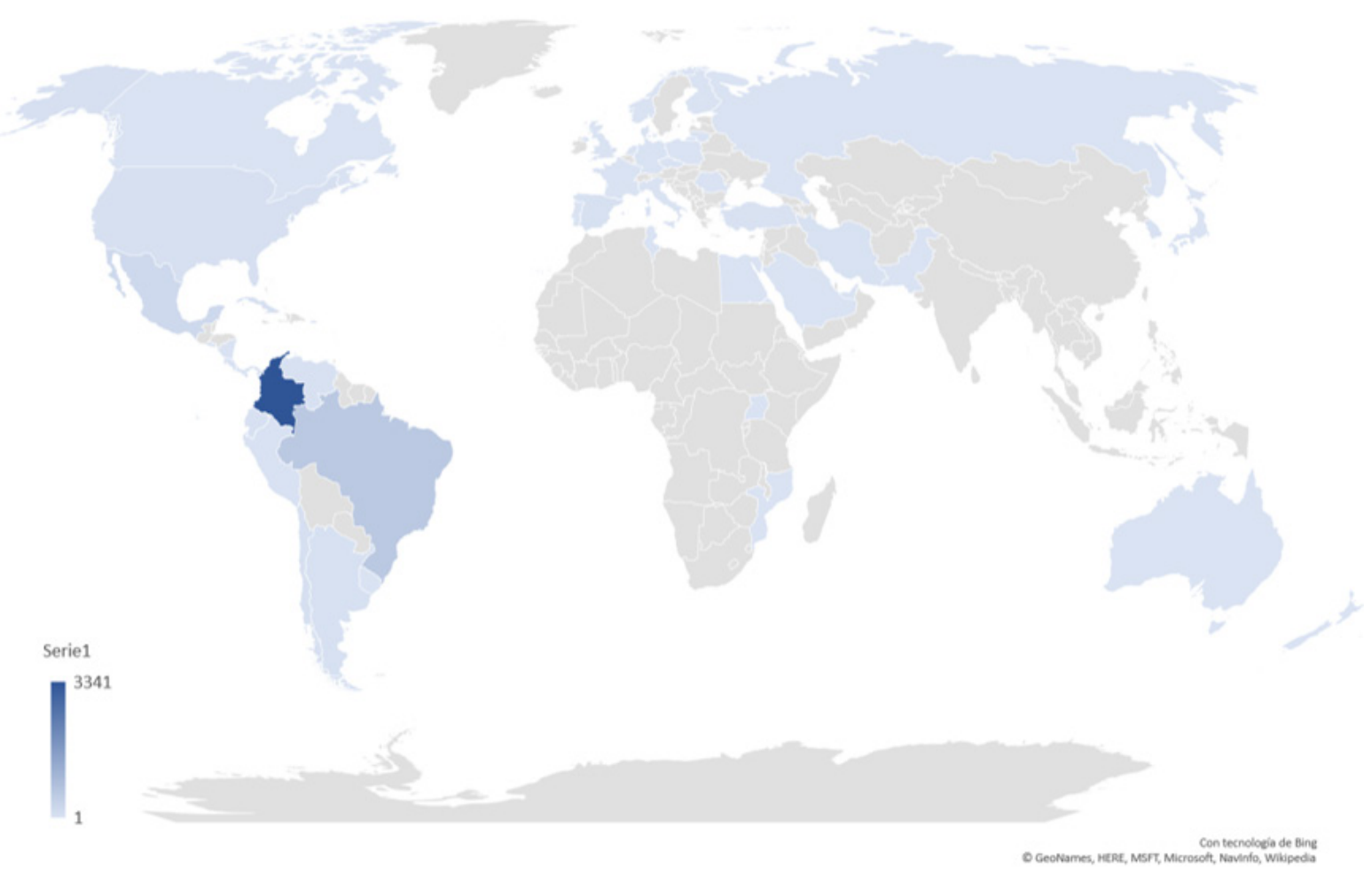

Figura 7. Geolocalización de la procedencia de autores de las revistas seleccionadas 
Para realizar la selección del listado de universidades, se tomaron las filiaciones de cada autor por cada revista y de ese listado, por conveniencia, debido al gran número de registros, se seleccionaron las 20 filiaciones de cada una de las revistas con más aparición en los registros, y luego, del total de estos registros, se realizó una consolidación de estos, con el fin de seleccionar las 25 filiaciones más representativas dentro del estudio (Tabla 3).

Tabla 3. Listado de filiaciones más citadas por los autores.

\begin{tabular}{|c|c|c|}
\hline Orden & Universidades & Registros \\
\hline 1 & Universidad Nacional de Colombia & 527 \\
\hline 2 & Universidad de Antioquia & 297 \\
\hline 3 & Universidad de Córdoba & 288 \\
\hline 4 & Universidad de Sucre & 237 \\
\hline 5 & Universidad de los Llanos & 215 \\
\hline 6 & Agrosavia & 191 \\
\hline 7 & Universidad de Ciencias Aplicadas y Ambientales & 134 \\
\hline 8 & Universidad de La Salle & 104 \\
\hline 9 & Universidad Pedagógica y Tecnológica de Colombia & 86 \\
\hline 10 & Universidad Estatal Paulista & 75 \\
\hline 11 & Universidad CES & 71 \\
\hline 12 & Universidad de Caldas & 70 \\
\hline 13 & Universidad de Nariño & 70 \\
\hline 14 & Universidad del Valle & 53 \\
\hline 15 & Universidad del Tolima & 48 \\
\hline 16 & Universidad de Cartagena & 42 \\
\hline 17 & Universidad Federal de Viçosa & 33 \\
\hline 18 & Corporación Universitaria Lasallista & 32 \\
\hline 19 & Pontificia Universidad Javeriana & 29 \\
\hline 20 & Práctica privada & 27 \\
\hline 21 & Instituto Nacional de Pesquisas da Amazônia & 20 \\
\hline 22 & Universidad de la Amazonía & 18 \\
\hline 23 & Universidad Cooperativa de Colombia & 15 \\
\hline 24 & Universidad de Sao Paulo & 15 \\
\hline 25 & Universidad Federal de Minas Gerais & 15 \\
\hline
\end{tabular}

Con relación a las áreas de publicación, zootecnia fue el área donde más se publicaron artículos, seguida de salud animal, agricultura, seguidas por otras áreas del conocimiento como se observa en la figura 8. Por otra parte, en la figura 9 se presenta una nube de palabras, incluyendo algunas subáreas relevantes dentro del estudio.

Se realizó una búsqueda individual por artículo en la plataforma de Google académico para identificar el número de citas de cada uno de los 3000 registros seleccionados. Se debe mencionar que esta plataforma puede tener algunas inconsistencias, pero fue utilizada por la disponibilidad de la información y aplicada a todas las revistas del estudio. La búsqueda se realizó en los dos idiomas del título de cada artículo y luego de sumaron las citas tanto en español como en inglés. En la tabla 4 se presentan cinco artículos representativos de cada una de las revistas estudiadas. 


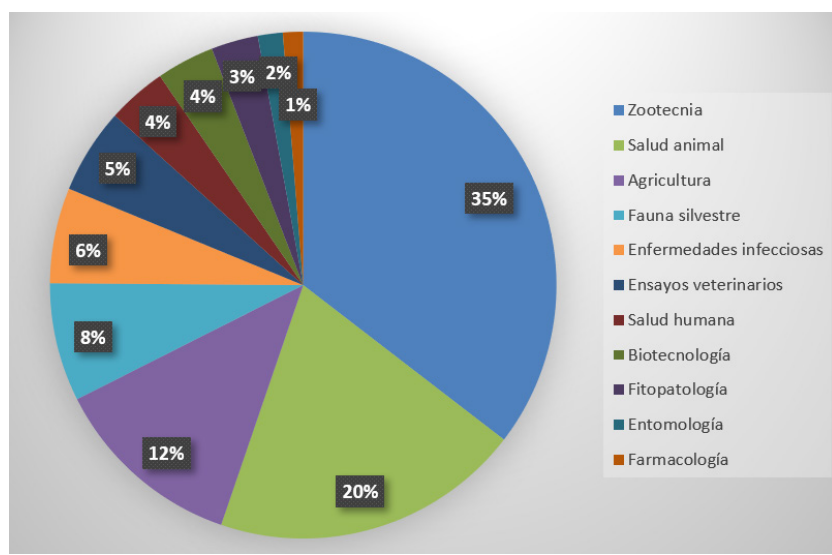

Figura 8. Áreas de estudio más publicadas en las revistas del estudio.

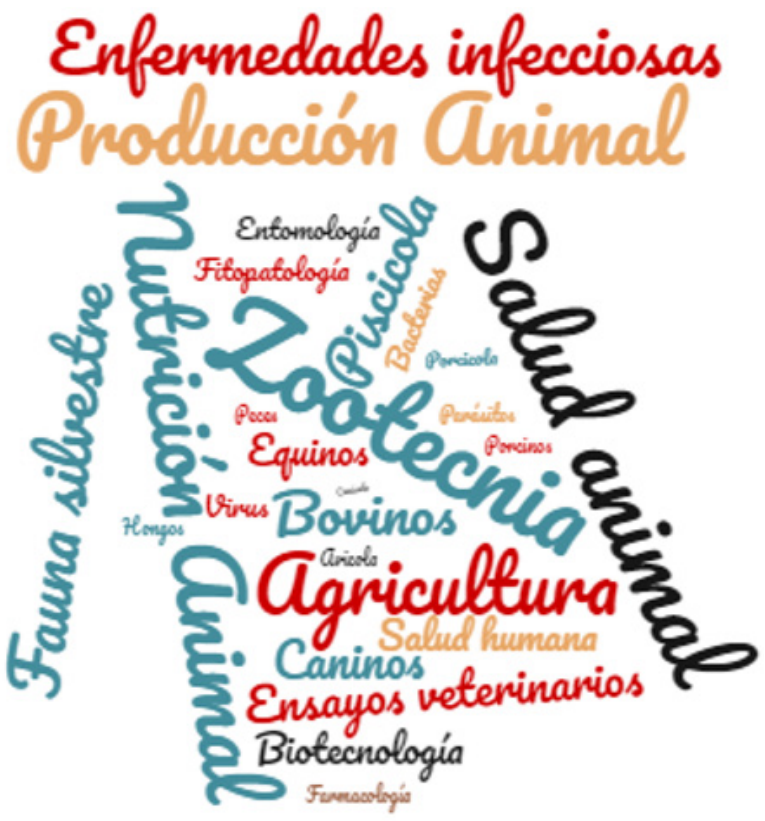

Figura 9. Nube de palabras de las áreas y subáreas del conocimiento más representativas de la investigación.

Tabla 4. Artículos más citados dentro del estudio.

\begin{tabular}{|c|c|c|c|}
\hline Rev & Nombre del artículo & \# Citas & URL \\
\hline \multirow{5}{*}{ 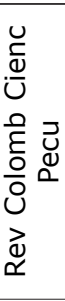 } & $\begin{array}{l}2014 \text { Contribution of intensive silvopastoral systems to animal performance and to adaptation and mitigation } \\
\text { of climate change }\end{array}$ & 71 & $\underline{U R L}$ \\
\hline & 2011 Prevalence of mastitis in dairy herds in Eastern Antioquia» & 45 & $\underline{\mathrm{URL}}$ \\
\hline & 2011 Dietary acidifiers in weanling pig diets: a review & 43 & $\underline{\mathrm{URL}}$ \\
\hline & 2011 Implicaciones de la inclusión del bienestar animal en la legislación sanitaria Colombiana & 33 & $\underline{\mathrm{URL}}$ \\
\hline & $\begin{array}{l}2011 \text { Prevalence of mastitis in dual purpose cattle farms in Monteria (Colombia): etiology and antibacterial } \\
\text { susceptibility }\end{array}$ & 32 & $\underline{\mathrm{URL}}$ \\
\hline \multirow{5}{*}{ 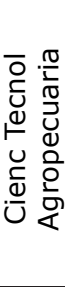 } & 2010 Microorganismos que mejoran el crecimiento de las plantas y la calidad de los suelos. Revisión & 80 & $\underline{\mathrm{DOI}}$ \\
\hline & 2011 Mecanismos de acción de las rizobacterias promotoras del crecimiento vegetal & 76 & $\underline{\mathrm{DOI}}$ \\
\hline & $\begin{array}{l}2010 \text { Distribución de garrapatas Rhipicephalus (Boophilus) microplus en bovinos y fincas del Altiplano } \\
\text { cundiboyacense (Colombia). }\end{array}$ & 61 & $\underline{\mathrm{DOI}}$ \\
\hline & 2014 La solubilización de fosfatos como estrategia microbiana para promover el crecimiento vegetal. & 60 & DOI \\
\hline & $\begin{array}{l}2010 \text { Cuantificación e interpolación de tendencias locales de temperatura y precipitación en zonas altoandinas } \\
\text { de Cundinamarca y Boyacá (Colombia). }\end{array}$ & 37 & $\underline{\mathrm{DOI}}$ \\
\hline \multirow{5}{*}{ 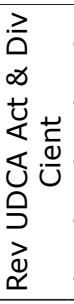 } & $\begin{array}{l}2011 \text { Interacción de microorganismos benéficos en plantas: Micorrizas, Trichoderma spp. y Pseudomonas } \\
\text { spp. Una revisión. }\end{array}$ & 88 & $\underline{\mathrm{URL}}$ \\
\hline & $\begin{array}{l}2010 \text { Evaluación in vitro del efecto antibacteriano de los extractos de Bidens pilosa, Lantana camara, Schinus } \\
\text { molle y Silybum marianum. }\end{array}$ & 64 & $\underline{\mathrm{URL}}$ \\
\hline & 2013 Reducción de la turbidez del agua usando coagulantes naturales: Una revisión. & 54 & $\underline{\mathrm{URL}}$ \\
\hline & $\begin{array}{l}2012 \text { Potencial de reutilización del efluente de la planta de tratamiento de aguas residuales de Nátaga en } \\
\text { cultivo de cacao (Theobroma cacao L.). }\end{array}$ & 52 & $\underline{\mathrm{URL}}$ \\
\hline & $\begin{array}{l}2011 \text { Nuevo método de ayuda diagnóstica con geometría fractal para células preneoplásicas del epitelio } \\
\text { escamoso cervical. }\end{array}$ & 42 & $\underline{\mathrm{URL}}$ \\
\hline \multirow{5}{*}{ 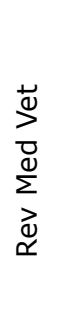 } & $\begin{array}{l}2010 \text { Importancia de los sistemas silvopastoriles en la reducción del estrés calórico en sistemas de producción } \\
\text { ganadera tropica.I }\end{array}$ & 62 & $\underline{\mathrm{DOI}}$ \\
\hline & $\begin{array}{l}2011 \text { Factores asociados a mastitis en vacas de la microcuenca lechera del altiplano norte de Antioquia, } \\
\text { Colombia }\end{array}$ & 48 & $\underline{\mathrm{DOI}}$ \\
\hline & $\begin{array}{l}\text { 2015 Seroprevalencia de Ehrlichia canis en perros con sospecha de infección por patógenos transmitidos } \\
\text { por garrapatas en Medellín, 2012-2014. }\end{array}$ & 27 & $\underline{\mathrm{DOI}}$ \\
\hline & $\begin{array}{l}\text { Gestión del conocimiento: mayor producción y competitividad. Perspectivas para los sistemas de } \\
\text { producción ovino-caprinos. }\end{array}$ & 26 & $\underline{\mathrm{DOI}}$ \\
\hline & 2011 Dirofilaria immitis: una zoonosis presente en el mundo & 24 & DOI \\
\hline
\end{tabular}


2011 Sobrepoblación canina y felina: tendencias y nuevas perspectivas.

2011 Evaluación del policultivo de bocachico Prochilodusmagdalenae y tilapia Oreochromis niloticus utilizando

2011 superficies fijadoras de perifiton.

$30 \underline{U R L}$

2014 Frecuencia de pythiosis cutánea en caballos de producción en explotaciones ganaderas de córdoba, $26 \quad$ DOI

2014 Determinación in vitro de la acción probiótica de Lactobacillus plantarum sobre Yersinia pseudotuberculosis aislada de Cavia porcellus.

2010 Purification, characterization and analysis of sepia melanin from commercial sepia ink (Sepia Officinalis).

2011 Nutritional characterization and ruminal degradation kinetics of some

N 2011 forages with potential for ruminant's supplementation in the highland tropics of Colombia.

2015 Monitoreo de Ehrlichia canis, Anaplasma phagocytophilum, Borrelia burgdorferi, y Dirofilaria immitis 2015 en perros de tres ciudades en Colombia.

2013 Evaluation of ryegrass (Lolium sp.) establishment in kikuyu grass 2013 (Pennisetum clandestinum) paddocks using zero tillage.

2013 Resistance of Salmonella to conventional antimicrobials for their treatment.

2010 Estructura y diversidad de bosques de galería en una sabana estacional de los Llanos Orientales

2010 colombianos (Reserva Tomo Grande, Vichada).

2010 La descomposición térmica de la cascarilla de arroz: Una alternativa de aprovechamiento integral.

2012 Perfil de los cuidadores informales de personas con enfermedades crónicas y calidad de vida,

2012 Villavicencio, Meta, 2011.

2012 Calidad higiénica y sanitaria de leche cruda acopiada en diferentes regiones colombianas.

\section{7}

2011 La palma de Moriche (Mauritia flexuosa L.f.) un ecosistema estratégico.

26

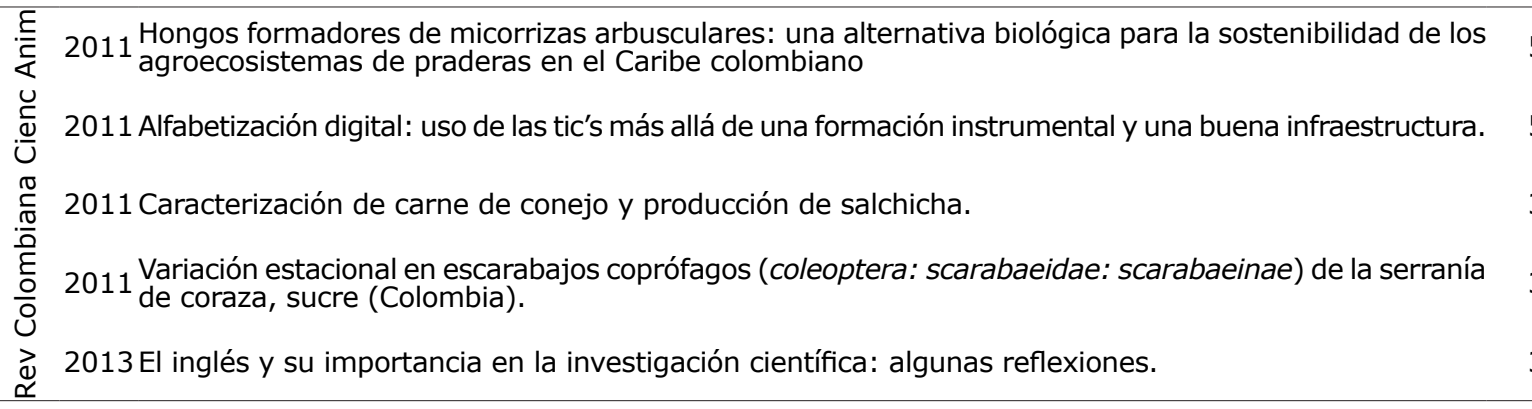

188 DOI

กำ

2014 Evaluation of the physicochemical and functional properties of Colombian bee pollen.

$50 \quad \underline{D O I}$

2012 Protozoan and metazoan parasites of Nile tilapia Oreochromis niloticus cultured in Brazil.

$43 \quad$ DOI

2011 Genetic diversity of six populations of red hybrid tilapia, using microsatellites genetic markers.

40

DOI

2011 Distribution of ectoparasites of Canis lupus familiarisL. (Carnivora: Canidae) from Panama.

34

$\underline{\text { DOI }}$

En la tabla 5 se observa el número total de citas que recibieron los cinco artículos mas citados. Se aprecia que la revista que recibió más citas fue la Revista MVZ Córdoba, seguida de la Revista Ciencia y Tecnología Agropecuaria, Revista
U.D.C.A. Actualidad \& Divulgación Científica, Revista Colombiana de Ciencias Pecuarias y Revista Colombiana de Ciencia Animal, por mencionar las del top 5. 
Tabla 5. Revistas con mayores citas provenientes sólo del top 5 de los arrticulos más citados en cada una de las revistas.

\begin{tabular}{lc}
\hline \multicolumn{1}{c}{ Revista } & Nro. de citas \\
\hline $\begin{array}{l}\text { Revista MVZ Córdoba } \\
\text { Revista Ciencia y Tecnología }\end{array}$ & 355 \\
$\begin{array}{l}\text { Agropecuaria } \\
\text { Revista U.D.C.A. Actualidad \& }\end{array}$ & 300 \\
$\begin{array}{l}\text { Divulgación Científica } \\
\text { Revista Colombiana de Ciencias } \\
\text { Pecuarias }\end{array}$ & 224 \\
$\begin{array}{l}\text { Revista Colombiana de Ciencia Animal } \\
\text { Revista CES Medicina Veterinaria y }\end{array}$ & 202 \\
$\begin{array}{l}\text { Zootecnia } \\
\text { Revista de Medicina Veterinaria }\end{array}$ & 198 \\
$\begin{array}{l}\text { Revista Orinoquia } \\
\text { Revista de la Facultad de Medicina } \\
\text { Veterinaria y de Zootecnia }\end{array}$ & 151 \\
\hline \multicolumn{2}{c}{ Total } \\
\hline
\end{tabular}

Se observa que en total estos artículos recibieron 2.092 citas, que resultan convenientes para las mismas revsitas y colateralmente tambien para el país.

\section{DISCUSIÓN}

En esta investigación se caracterizó la producción científica de las principales revistas del área de ciencias veterinarias en el período 20102019. Los resultados obtenidos permitieron el diagnóstico general de las revistas involucradas, pero es necesario aclarar que el análisis realizado, no permite evaluar la calidad de las revistas o de los artículos publicados en las mismas, sino realizar un análisis de los artículos, recurriendo a los índices empleados en el ámbito internacional para evaluar las publicaciones científicas.

La importancia de estos estudios de bibliometría se basan en la posibilidad de identificar las fortalezas y las debilidades de las publicaciones, pero siempre bajo una mirada constructiva orientada a proporcionar los elementos necesarios a los editores que le permitan proponer estrategias para incrementar el nivel de competencia, posicionamiento y rigor exigido por la comunidad científica internacional de las revistas que dirigen.
En la presente investigación se analizaron las principales características de la producción científica entre 2010 y 2019 en las revistas colombianas que tienen en común el area de Medicina Veterinaria y Zootecnia. Los resultados permitieron observar por primera vez y con algún detalle cuál ha sido la actividad investigadora de esta área en Colombia.

Dentro del tipo de documentos considerasdos en el estudio, la mayor producción correspondió a los artículos originales producto de investigación. Este hallazgo permitió inferir que se está realizando un aporte significativo al nuevo conocimiento en el área de ciencias veterinarias en Colombia. De este aporte debe destacarse que más de $80 \%$ lo hacen las universidades públicas y el resto las privadas. Estos resultados coinciden con las apreciaciones hechas por Bravo-Vinaja y Sanz-Casado (18) que informan que la actividad investigadora es mayor en las universidades públicas y los institutos o centros de investigación. Estas proporciones pueden variar de acuerdo con la temática estudiada, tal como lo reportan Ríos et al (20) que afirman que la universidad pública soporta el $90 \%$ de la investigación del área de infectología en Colombia.

Bravo-Vinaja y Sanz-Casado (18) también informan que existe una alta concentración de la producción científica proveniente de los grandes centros urbanos y particularmente en dos de los estados mexicanos más desarrollados y poblados. Algo similar también fue observado en Colombia por Ríos et al (20) quienes comentan que quizás tenga alguna relación con la mayor concentración laboral y por tanto residencial de la masa crítica de investigadores en los grandes centros urbanos, o que a su vez está en consonancia con el tamaño de las instituciones de investigación o de las mismas universidades.

En este contexto, Herrán-Páez (21), reportan que para el período 2003-2015 las instituciones de educación superior fueron las que aportaron el mayor número de artículos con $41 \%$ de la producción total del país en contraste con la obtenida en este estudio que fue superior al $80 \%$. Este incremento quizás tenga que ver con las políticas del Ministerio de Ciencias, tecnología e innovación mediante la financiación de la investigación atendiendo las convocatorias que sobre la materia abren anualmente, así como los continuos estímulos a los docentes investigadores para que produzcan nuevo conocimiento. La relativamente alta producción 
científica colombiana en el área de ciencias veterinarias se ve reflejada con la publicación de artículos originales ( $76.1 \%)$ que son producto de la investigación. También es importante mencionar la producción en las otras modalidades consideradas en este estudio que fueron importantes y que correspondieron a revisiones de literatura, casos clínicos, comunicaciones breves y editoriales.

El idioma que predominó en las publicaciones fue el español (75\%), le siguió el inglés (22.4\%) y por último el portugués (2.6\%). En contraste con los resultados de Bravo-Vinaja y Sanz-Casado (18) en su estudio realizado en el Distrito Federal y en el Estado de México, el idioma de publicación predomínate fue el inglés, seguido del español.

Analizando ligeramente el efecto indirecto que podría tener el idioma de publicación, se observa que las revistas que publican en inglés o español e inglés, como son la Revista Colombiana de Ciencias Pecuarias y la Revista MVZ Córdoba, ostentan un mejor posicionamiento en los rankings internacionales; ambas clasificadas en el cuartil 3 (Q3) de SCOPUS. Igualmente son las revistas que tienen mayor colaboración internacional, lo que indiscutiblemente favorece la visibilidad internacional, no sólo de las revistas "per se", sino de Colombia.

Los autores nacionales prevalecieron en las revistas analizadas, por tanto se requiere de un mayor esfuerzo para traspasar la frontera y llevar las revistas a un contexto internacional con el fin de difundir sus contenidos en bases de datos e índices internacionales, lo que probablemente permita interesar a un mayor número de autores extranjeros y así contribuir con la superación de las barreras regional y nacional. Por esa razón creemos que una de las estrategias para logralo, es realizar las publicaciones en inglés, o como en el caso de la Revista MVZ Córdoba, publicar los contenidos en español e inglés. Estos resultados son similares a los reportados por Maz-Machado et al (25) en un estudio de la producción científica colombiana.

La colaboración de autoría, también llamado índice de colaboración puede interpretarse como resultado de la interacción de equipos de trabajo, aspecto que en la actualidad es de suma importancia para la consecución de recursos para investigación en entidades gubernamentales e internacionales. En estas convocatorias se examina en detalle la participación interdisciplinaria e interinstitucional, incluso entre instituciones públicas, privadas y entes territoriales. Por esta multi y pluriparticipación de autores en la investigación actual, creemos que el número de autores por artículo, lo más probable es que tienda al incremento en los próximos años.

En el presente estudio el promedio de autores por artículo fue de 3.38 , lo que podría considerase como bajo si se tienen en cuenta los conceptos antes referidos como son interdisciplinariedad e interinstitucionalidad. Este resultado, quizás esté influenciado de alguna manera en la inclusión de autores en entidades públicas, ya que, en estas, a mayor número de autores por artículo, los estímulos se disminuyen porque se dividen con un mayor número de interesados, mientras que, en las entidades privadas, este no ocurre. Por su parte, Bravo-Vinaja y Sanz-Casado (18) informan una tasa media de artículos firmados en coautoría de $87.62 \%$, pero en contraste, el índice de coautoría aumentó de 2.47 autores por artículo desde 1983 a 4.08 para 2002.

De todas maneras, creemos que la tendencia de colaboración debe aumentar y, por tanto, convendría la promulgación de estas políticas por parte de las instituciones gubernamentales, así como aquellas relacionadas con la financiación de la investigación. Para mejorar la coautoría también pueden ser beneficiosas la creación de redes académicas tanto nacionales como internacionales. La instauración de estas estrategias muy probablemente producirá un mejoramiento en la mirada internacional de las revistas colombianas por parte de potenciales autores y lectores.

En un estudio sobre las publicaciones relacionadas con las enfermedades infecciosas en Colombia (2000-2009), Ríos et al (20) contabilizaron 2963 publicaciones, 2744 (92.6\%) fueron nacionales y 219 ( $7.4 \%)$ fueron extranjeras. En el presente estudio se contabilizaron 10.296 autores que publicaron en ciencias veterinarias, de ellos, 7.109 (69\%), correspondieron a autores nacionales y 3.187 (31\%) a extranjeros, notándose un importante incremento de la participación de autores extranjeros en este estudio. Dando una mirada general, estos resultados significan que la mayoría de las revistas colombianas son utilizadas por autores nacionales para la publicación de sus investigaciones. En contraste, se observa que en la Revista MVZ Córdoba y la Revista Colombiana de Ciencias Pecuarias, publican más autores extranjeros que nacionales. Este resultado, como 
se ha mencionado, quizás esté relacionado con la publicación de estas dos revistas en idioma inglés, lo que muy seguramente atrae más lectores en el ámbito global.

Con relación al género de los autores, se encontró que 6.659 (64.7\%) fueron hombres; 3.199 $(31 \%)$ mujeres y $438(4.3 \%)$ no fue posible identificar a través de su firma en los artículos. Se observa que la participación masculina dobla la femenina, lo que sugiere un desequilibrio de géneros en la investigación, como lo ha comentado Menéndez (26). Los resultados del presente estudio son similares a los informados por Larivière et al (27), quienes hallaron que las mujeres representan globalmente menos del $30 \%$ de las autorías compartidas, en contraste con los hombres que alcanza el $70 \%$. En otras palabras, por cada artículo que una mujer firme como primera autora, hay cerca de dos trabajos (1.93) firmados por un hombre. Afirman también que estas diferencias se acentúan en Sudamérica y Europa del este. La importancia del trabajo de Larivière et al (27), fue que incluyó 5.4 millones de trabajos de investigación y revisiones de literatura a nivel global; incluyendo 27.3 millones de autores entre los años 2008 y 2012.

A nuestro modo de ver la situación global, así como la promulgación de las políticas de inclusión en el ámbito internacional, creemos que esta brecha de participación en la investigación de mujeres versus hombres tendrá la tendencia a cerrarse, en especial si se considera el incremento sostenido de la participación de la mujer en todos los campos laborales productivos y de los saberes, y la investigación, nos es la excepción.

Respecto a la geolocalización de las consultas de las revistas colombiana de ciencias veterinarias se observó que las consultas pueden considerarse que las realizan en el ámbito global, con muy pocas o nulas consultas en algunos casos en Africa, Asía meridional y Groenlandia.

Con relación a las cinco universidades que más filiaciones contabilizaron y por tanto, las de mayor producción fueron la Univesidad Nacional de Colombia, Universidad de Antioquia, Universidad de Córdoba, Universidad de Sucre y la Universidad de los Llanos. Estos resultados concuerdan parcialmente con los obtenidos por Máttar et al (22) quienes encontraron que las universidades colombianas con mayor productividad fueron: Nacional de Colombia, Antioquia, Andes, Valle y Javeriana. En la región
Caribe fueron: Norte, Cartagena, Córdoba, Magdalena y Sucre. Según estos autores (22), con los que estamos de acuerdo, este tipo de estudios facilitan dar una mirada contextualizada de la producción científica y, recomiendan que las IES debieran apostarle a la calidad y, utilizar, al menos, los cinco factores que utiliza SCImago. Aunque los rankings no son obligatorios, ignorarlos significaría autoaislamiento, lo que, a nuestro juicio, no sería nada conveniente en este mundo tan globalizado.

Las áreas de mayor publicación obtenidas en este estudio fueron, zootecnia $(35 \%)$, salud animal (12\%), agricultura (12\%), fauna silvestre $(8 \%)$, enfermedades infecciosas $(6 \%)$, ensayos veterinarios $(5 \%)$, salud humana $(4 \%)$ y biotecnología (4\%). Por otra parte, Llalla et al (17) en un estudio realizado en la facultad de medicina veterinaria y zootecnia de la universidad peruana Cayetano Heredia, encontraron que los animales domésticos fueron los más estudiados $(43.6 \%)$, seguidos de los silvestres $(30.3 \%)$; los caninos $(29.2 \%)$ y el hombre $(15.4 \%)$ fueron las especies más estudiadas. Es necesario aclarar que los estudios vinculados con el hombre estuvieron relacionados con las enfermedades zoonóticas, la identificación de conocimientos y prácticas riesgosas en la relación humano-animal, así como las consecuencias de no observar las medidas de prevención de las zoonosis; información importante para los profesionales encargados la salud pública. En contraste, Rodríguez-Ledesma et al (19), centraron su investigación en áreas predeterminadas como: Alimentación de animales, pequeños rumiantes, reproducción animal, producción láctea, calidad de carne, producción porcina, cría genética y animal, aves de corral, bienestar animal y factores de crecimiento. Pero también se ha orientado al bienestar animal, a la genómica, a la gestión y a la salud humana. Como se observa, estas comparaciones son difíciles de hacer, pues cada autor orienta su investigación de acuerdo con su interés particular y por tanto respetables y finalmente aceptables.

Finalmente hay que centrarse que lo que incrementa el prestigio de las revistas en la actualidad, es la citación que reciben sus artículos, tal como lo utilizan los rankings internacionales con defensores y detractores sobre esta clasificación y metodología.

No obstante, las mediciones bibliométricas pueden ser insuficientes si se utilizan de manera aislada como medidas de calidad e importancia 
de la investigación. Las herramientas que vinculan las revisiones por expertos de la calidad y de la importancia de la investigación en particular con indicadores cuantitativos, como el análisis de citas, por ejemplo, son un valioso instrumento adicional que no se puede olvidar en la evaluación de la investigación (10).

Se concluye que las revistas que publican en inglés consiguen mejor posicionamiento internacional y mayor número de autores extranjeros. El tipo de artículos que más se publicó en las revistas colombianas analizadas fueron los artículos originales y el número promedio de autores por artículo fue de tres. La mayor producción científica corresponde a la universidad pública ( $\geq 80 \%$ ) y los artículos son consultados globalmente, pero con menores o nulas consultas en África, Asía meridional y Groenlandia. El top 5 de las filiaciones de autores correspondió a universidades públicas y las áreas más estudiadas fueron zootecnia, salud animal, agricultura y fauna silvestre. El top 10 de los artículos más citados arrojó un total de 810 citas que contribuyen con la internacionalización de las revistas y de Colombia.

\section{Conflicto de intereses}

Los autores de este artículo no tenemos ningún conflicto de intereses en la elaboración, producción y publicación de este manuscrito.

\section{Participación de los autores en el desarrollo del manuscrito}

Los autores firmantes declaramos que tuvimos participación en la formulación de la propuesta, obtención de la información, análisis de esta y escritura del manuscrito. La escritura de este manuscrito fue parte de los requisitos para optar el grado de Especialistas en Edición de Publicaciones, perteneciente a la Escuela Interamericana de Bibliotecología de la Universidad de Antioquia. Medellín, Colombia.

\section{Agradecimientos}

A la Universidad de Antioquia, a la Escuela Interamericana de Bibliotecología, a los compañeros de curso, a los profesores y directivos (as) de la especialización, al Dr. Alejandro Uribe Tirado como tutor y a la Universidad de Córdoba y Facultad de Medicina Veterinaria y Zootecnia.

\section{REFERENCIAS}

1. Abadal E. Revistas científicas. Situación actual y retos de futuro. Edicions Universitat Barcelona: Barcelona, España; 2017. https://tinyurl.com/y5t4oes8

2. López-Ornelas M, Cordero-Arroyo G. "Un intento por definir las características generales de las revistas académicas electrónicas". Revista Razón y Palabra. 2005; 10(43):1-33. http://hdl.handle. net/10760/15700

3. Castillo-Esparcia A, Rubio-Moraga Á, Almansa-Martínez $A$. La investigación en comunicación. Análisis bibliométrico de las revistas de mayor impacto del ISI. Revista Latina de Comunicación Social. 2012; 67:248-270. https://doi.org/10.4185/ RLCS-067-955-248-270.
4. Hood WW, Concepción SW. The literature of bibliometrics, scientometrics, and informetrics. Scientometrics. 2001; 92(2):291-314. https://doi. org/10.1023/A:1017919924342

5. Alonso Arévalo J, Cordón-Garcia JA, Maltrás Barba B. Altmetrics: medición de la influencia de los medios en el impacto social de la investigación. Cua Doc Multimedia. 2016; 27:75-101. https://doi.org/10.5209/ rev CDMU.2016.v27.n1.52870

6. Allen L, Jones C, Dolby K, Lynn D, Walport M. Looking for Landmarks: The Role of Expert Review and Bibliometric Analysis in Evaluating Scientific Publication Outputs. PLoS ONE. 2009; 4(6):e5910. https://doi. org/10.1371/journal.pone.0005910 
7. Sanz-Valero J, Tomás Casterá V, WandenBerghe $C$. Estudio bibliométrico de la producción científica publicada por la Revista Panamericana de Salud Pública/ Pan American Journal of Public Health en el período de 1997 a 2012. Rev Panam Salud Publica. 2014; 35(2):81-8. http://iris.paho. org/xmlui/handle/123456789/8465

8. Garfield E. Citation indexes to science: a new dimension in documentation through association of ideas. Science. 1955; 123(3159):108-111. http://dx.doi. org/10.1126/science.122.3159.108

9. Smith DR. Impact factors, scientometrics and the history of citation-based research Scientometrics. 2012; 92:419. https://doi. org/10.1007/s11192-012-0685-X _

10. Cobo MJ, Martínez MA, Gutiérrez-Salcedo M, Fujita $H$, Herrera-Viedma E. 25 years at knowledge-based systems: a bibliometric analysis. Knowl Based Syst. 2015; 80:3-13. http://dx.doi.org/10.1016/j. knosys.2014.12.035

11. Priem J, Taraborelli D, Groth P, Neylon C. Altmetrics: A Manifesto. [Accessed March 21 2020]. 2010. http://altmetrics.org/ manifesto/.

12. Priem J, Groth P, Taraborelli D. The Altmetrics Collection. PLoS ONE 2012; 7(11):e48753. http://dx.doi.org/10.1371/ journal.pone. 0048753

13. Melero R. Altmetrics - a complement to conventional metrics, Biochemia Medica. 2015; 25(2):152-160. http://dx.doi. org/10.11613/BM.2015.016.

14. Repiso, R., Castillo-Esparcia, A. y TorresSalinas, D. Altmetrics, indicadores alternativos para revistas de estudios de comunicación de la Web of Science. Scientometrics. 2019; 119:941-958. https://doi.org/10.1007/s11192-019$\underline{03070-7}$

15. Hicksa D, Woutersb P, Waltmanb $L$, de Rijckeb S, Rafols I. The Leiden Manifesto for research metrics. Nature. 2015; 520:429431. https://www.nature.com/news/ bibliometrics-the-leiden-manifesto-forresearch-metrics-1.17351
16. Crawley -Low J. Bibliometric analysis of the American Journal of Veterinary Research to produce a list of core veterinary medicine journals. J Med Libr Assoc. 2006; 94(4):430434. https://www.ncbi.nlm.nih.gov/pmc/ articles/PMC1629416/

17. Llalla VT, Mendoza TG, Falcón PN. Evaluación bibliométrica de la investigación formativa en la Facultad de Medicina Veterinaria y Zootecnia de la Universidad Peruana Cayetano Heredia en el período 2012-2017. Salud Tecnol Vet. 2018; 6(1):9-19. https:// doi.org/10.20453/stv.v6i1.3373

18. Bravo-Vinaja Á, Sanz-Casado E. Análisis bibliométrico de la producción científica de México en Ciencias Agrícolas durante el período 1983-2002. Revista Fitotec Mex. 2008; 31(3):187-194. https:// www.revistafitotecniamexicana.org/ documentos/31-3/1a.pdf

19. Rodriguez-Ledesma A, Cobo MJ, LopezPujalte C, Herrera-Viedma E. An overview of animal science research 1945-2011 through science mapping analysis. J Anim Breed Genet. 2015; 132(6):475-497. https://doi.org/10.1111/jbg.12124

20. Ríos R, Mattar S, González M. Análisis bibliométrico de las publicaciones sobre enfermedades infecciosas en Colombia, 2000-2009. Rev Salud Pública. 2011; 13(2): 98-307. https://revistas.unal.edu. co/index.php/revsaludpublica/article/ view/15599/38078

21. Herrán-Páez E. Análisis bibliométrico de la producción científica colombiana (2003-2015). Granada, España: Ediciones Profesionales de la Información; 2019. https://doi.org/10.3145/colombia scimago

22. Mattar VS, González TM, Salgado-Arroyo L. Análisis de las universidades colombianas de acuerdo con el ranking SCImago 20102012. Rev MVZ Córdoba. 2013; 18(1):33993407. https://doi.org/10.21897/rmvz.203

23. Carreño LM, Poutou-Piñales RA, Mattar S, González M. Indicadores bibliométricos de actividad de la Revista MVZ Córdoba 19942008. Rev MVZ Córdoba. 2009; 14(1):15311543. https://doi.org/10.21897/rmvz.363 
24. De La Ossa J, Montes-Vergara D, González TM, Salgado AL. Análisis bibliométrico de la Revista Colombiana de Ciencia Animal - RECIA 2009-2018. Indicadores de producción. Rev Colombiana Cienc Anim. Recia. 2019; 11(1):Artículo724. https://doi. org/10.24188/recia.v11.n1.2019.724

25. Maz-Machado A, Jiménez-Fanjul N, Villarraga M. La producción científica colombiana en SciELO: un análisis bibliométrico. Rev Interam Bibliot. 2016; 39(2):111119. https://doi.org/10.17533/udea.rib. $\mathrm{v} 39 \mathrm{n} 2 \mathrm{a} 03$
26. Clara Menendez. El papel de la mujer en la investigación científica y médica en el siglo xxi: un debate necesario. Atención Primaria. 2011; 43(7):331-332. https:// doi.org/10.1016/j.aprim.2011.06.001

27. Larivière $\mathrm{V}, \mathrm{Ni} \mathrm{C}$, Gingras $\mathrm{Y}$, Cronin $\mathrm{B}$, Sugimoto CR. Bibliometrics: Global gender disparities in science. Nature. 2013; 504(7479):211-213. https://doi. org/10.1038/504211a 